\title{
Critical slowing-down as indicator of approach to the loss of stability
}

\author{
Dmitry Podolsky \\ Department of Mechanical Engineering, \\ Massachusetts Institute of Technology \\ Cambridge, MA, 02139 \\ Email: podolsky@mit.edu
}

\author{
Konstantin Turitsyn \\ Department of Mechanical Engineering, \\ Massachusetts Institute of Technology \\ Cambridge, MA, 02139 \\ Email: turitsyn@mit.edu
}

\begin{abstract}
We consider stochastic electro-mechanical dynamics of an overdamped power system in the vicinity of the saddlenode bifurcation associated with the loss of global stability such as voltage collapse or phase angle instability. Fluctuations of the system state vector are driven by random variations of loads and intermittent renewable generation. In the vicinity of collapse the power system experiences so-called phenomenon of critical slowing-down characterized by slowing and simultaneous amplification of the system state vector fluctuations. In generic case of a co-dimension 1 bifurcation corresponding to the threshold of instability it is possible to extract a single mode of the system state vector responsible for this phenomenon. We characterize stochastic fluctuations of the system state vector using the formal perturbative expansion over the lowest (real) eigenvalue of the system power flow Jacobian and verify the resulting expressions for correlation functions of the state vector by direct numerical simulations. We conclude that the onset of critical slowing-down is a good marker of approach to the threshold of global instability. It can be straightforwardly detected from the analysis of singlenode autostructure and autocorrelation functions of system state variables and thus does not require full observability of the grid.
\end{abstract}

\section{INTRODUCTION}

Most of the US national power grid has ultimately been shaped and built during the quick urbanization period of the beginning of $20^{\text {th }}$ century. Increasing power demand continues to put enormous strain on the infrastructure of the aging grid, forcing utilities to maximally utilize its existing resources. As a consequence, many parts of the grid often operate in nearly critical regimes with significantly increased probability of large scale failures [1], [2], [3]. In the modern world it becomes crucially important to be able to read the state of the pre-critical smart grid in a timely and precise manner, to effectively control parameters of smart grids operating in precritical regimes minimizing the probability of their large scale failures. Unfortunately, commonly used used power system state estimation and control algorithms, working extremely well for stable operating regimes, become less efficient (see for example [4], [5]) when the operating point of the grid is close to the threshold of instability.

As we shall discuss below, a partial reason for the decrease of efficiency of state estimation procedures is related to a strong amplification of fluctuations of the power system state vector closer to the threshold of instability. The present contribution addresses the problem of stochastic fluctuations of power system state vector close to voltage or phase-angle instabilities. ${ }^{1}$

The amplification of stochastic fluctuations of the system state vector in pre-critical operating regimes is also directly related to the phenomenon of critical slowing-down often observed in power grids on the brink of failure [11], [12], [13], [14]. Naturally, detecting the onset of critical slowingdown by itself can provide an efficient way for early detection of approach to a large-scale instability [11], [12], [13], [14]. As will be explained below, the onset of critical slowing-down is naturally associated with a strong growth of fluctuations of the system state vector. This growth can in turn be locally identified well befiore the event of collapse from local synchrophasor measurements of voltage phase and magnitude on a given node of the smart grid.

The contributions of our work can be summarized as follows. We provide a formal mathematical description of critical slowing-down phenomenon, and characterize it in terms of power flow Jacobian as well as generator inertia and damping matrices. Second, we derive a closed form equation for the autocorrelation and autostructure functions as well as power spectral density of state vector that completely characterizes the probabilities of arbitrary system trajectories. Remarkably, the autocorrelation and autostructure functions are expressed in terms of steady-state power flow Jacobian and its eigenvectors. Finally, we validate all our results with numerical simulation of IEEE 39 and IEEE 57 test systems.

\section{POWER FLOW AND LOAD MODELS}

To describe dynamics of system variables close to the threshold of instability, we use the structure-preserving model [15] which reduces to the system of coupled swing equations on $(P, V)$ (generator) nodes of the power grid

$$
\frac{H_{i}}{\pi f_{0}} \frac{d^{2} \theta_{i}}{d t^{2}}+\alpha_{i} \frac{d \theta_{i}}{d t}=\sum_{j \sim i} \mathcal{Y}_{i j} V_{i} V_{j} \sin \left(\theta_{i}-\theta_{j}-\gamma_{i j}\right)+P_{m, i}
$$

${ }^{1}$ The stochastic models of power system dynamics have been studied before in a number of papers, such as [6], [7], [8], [9], [10] 
and power flow equations on $(P, Q)$ (consumer) nodes of the grid

$$
\begin{aligned}
P_{i} \approx & P_{0, i}+\alpha_{p, i} \dot{\theta}_{i}+\beta_{p, i}\left(V_{i}-V_{0}\right)+T_{p, i} \dot{V}_{i}= \\
& =\sum_{j \sim i} \mathcal{Y}_{i j} V_{i} V_{j} \sin \left(\theta_{i}-\theta_{j}-\gamma_{i j}\right), \\
Q_{i} \approx & Q_{0, i}+\alpha_{q, i} \dot{\theta}_{i}+\beta_{q, i}\left(V_{i}-V_{0}\right)+T_{q, i} \dot{V}_{i}= \\
= & \sum_{j \sim i} \mathcal{Y}_{i j} V_{i} V_{j} \cos \left(\theta_{i}-\theta_{j}-\gamma_{i j}\right) .
\end{aligned}
$$

Here $\theta_{i}$ is a voltage phase on a bus $i, H_{i}$ is inertia constant of a generator on the node $i$, parameters $\alpha_{i}$ describe droop controls of generators and/or load dependence on frequency fluctuations $\omega_{i}=\dot{\theta}_{i}$ on the $(P, Q)$ nodes, $V_{i}$ is a voltage magnitude on a bus $i$ (for the $(P, V)$ nodes, $V_{i}=E_{i}$ ), parameters $\beta$ encode dependence of the power load on the voltage magnitude $V_{i}$. Finally, coefficients $T_{i}$ describe a (weak) dependence of the power load on the rate $\dot{V}_{i}$ of the voltage change with time. The power losses are important for the dynamics of system state variables, thus generally $\gamma_{i j} \neq 0$.

We consider the load model (2), (3) with such parameters chosen that $\beta_{i}=0$ for simplicity, the choice of $\alpha_{i}$ corresponds to $1 \div 2 \%$ change of the load per $1 \%$ change in system frequency $f$ and $10 \div 20 \%$ change in power generation per $1 \%$ change in $f[16]$, while parameters $T_{i}$ - to $0.1 \%$ change in power load per $1 \%$ change in $\dot{V}$. For simplicity we assume, that all the loads have fixed power factors $P F_{i}=k_{i} / \sqrt{1+k_{i}^{2}}$, and all fluctuate with time in the vicinity of the average (base) load $\bar{P}_{i}(t)$, constantly deviating from the base value and returning back, so that $P_{i}(t)=\bar{P}_{i}(t)+\delta P_{i}(t), Q_{i}(t)=$ $\bar{Q}_{i}(t)+\delta Q_{i}(t)$ [6], [7]. The same applies to generated power, especially if the grid is exposed to intermittence of renewable energy sources. Thus, $P_{i}$ generally behave as stochastic processes [17]. The base load value $\bar{P}_{i}(t)$ itself changes with time but relatively slowly, with significant changes only noticeable at time scales of several hours. In principle, a given, aggregated load $P_{i}(t)$ can be thought of as an aggregation of a huge number of power-consuming devices connected to the node $i$ of the grid, which get online and offline, connected and disconnected from the grid. A resulting overall time profile of the aggregated active load $P_{i}(t)$ is represented on the Fig. 1. The characteristic time scale $t_{\text {on/off }}$ of step-like changes depicted there is $0.1-1 \mathrm{sec}$. These relatively fast power load fluctuations on different nodes of the grid are statistically independent from each other, and their correlation properties of the vectors $\delta P, \delta Q$ can be considered Gaussian [17]. The latter have mean zero and are thus completely characterized by the expectation values

$$
E\left(\delta P(t) \delta P^{T}\left(t^{\prime}\right)\right)=\mathcal{B}\left(t-t^{\prime}\right),
$$

while

$$
E\left(\delta Q(t) \delta Q^{T}\left(t^{\prime}\right)\right)=k \mathcal{B}\left(t-t^{\prime}\right) k^{T},
$$

and

$$
E\left(\delta P(t) \delta Q^{T}\left(t^{\prime}\right)\right)=k \mathcal{B}\left(t-t^{\prime}\right)
$$
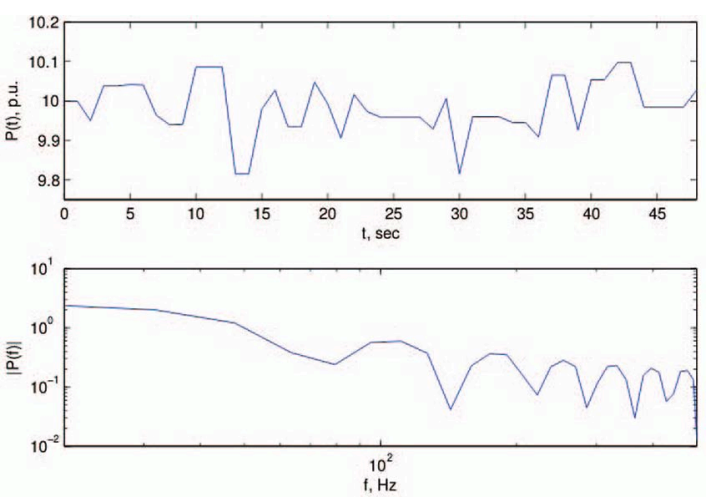

Fig. 1. Time profile of aggregated power load $P_{i}(t)$ and the absolute value of its Fourier transform.

here $\mathcal{B}\left(t-t^{\prime}\right)$ is a diagonal covariance matrix. Note that generally $\mathcal{B}\left(t-t^{\prime}\right) \neq$ Const. Alternatively, the fluctuations can be represented with the power spectral density $E\left(\delta P(f) \delta P^{T}(-f)\right)$, which is simply a Fourier transform of the autocorrelation function (4), see for example [17]. Here and in the forthcoming paragraphs the averaging corresponds to summation over possible realization of the load fluctuations. For stationary stochastic processes, the same results could be recovered by averaging over long time periods $T$.

Whenever the random load profile is composed of a continuing sequence of independent individual "jumps", the power spectral density $\mathcal{B}(f)$ behaves like $\mathcal{B}_{0} /\left((2 \pi f)^{2}+\tau^{-2}\right)$, where $\tau$ is the characteristic time of power load correlations. We assume that this time is much longer than the typical timescales of $1-30 \mathrm{~s}$ that are considered in this work, but smaller than the scale of several hours. On the time scales correspoding to large enough frequencies, such that $f \gg \tau^{-1}$, one approximately has $\mathcal{B}(f)=\mathcal{B}_{0} /(2 \pi f)^{2}$. The same approximation has been also proposed and justified in [18]. Importantly, this model is different from the ones considered in a number of other studies that rely on a more traditional "white noise" model [10], [14], [19]. The latter implies that the correlation properties of the fluctuating power loads are described by $E(\delta P(f) \delta P(-f)) \approx$ $\mathcal{B}_{0}$, independent of $f$. The power of such fluctuations of $\delta P$ does not diminish with frequency, which is not realistic.

Close to the equilibrium operating point, the nonlinear system of equations (1), (2), (3) can be linearized:

$$
\mathcal{M} \ddot{x}+\mathcal{D} \dot{x}+\mathcal{K} x=\delta P,
$$

where $x$ is the system state vector respreseting the deviations of voltage phases and magnitudes from the equilibrium value, $\mathcal{K}$ is the power flow Jacobian, the diagonal matrix $\mathcal{M}$ encodes inertia of the turbines, while the diagonal matrix $\mathcal{D}$ corresponds to the frequency damping. The system (5) of Stochastic Differential Equations (SDE in what follows) will be the main subject of our study. 


\section{STOCHASTIC DYNAMICS OF SYSTEM VARIABLES NEAR THE THRESHOLD OF INSTABILITY: THEORETICAL DESCRIPTION}

We discuss the general case of a power grid with a large number of $(P, V)$ nodes (generators) and $(P, Q)$ nodes (loads). It is natural to assume that the graph of the grid under consideration does not have any specific translational/rotational symmetries, in which case the saddle-node bifurcation, corresponding to the global collapse of the power system, has a co-dimension 1. The autocorrelation function $C(\delta t)=$ $E\left(\left(x(t+\delta t) x^{T}(t)\right)\right.$ of the system state vector $x$ is given by

$$
C(|\delta t|)=\int d f e^{-j 2 \pi f|\delta t|} C(f)
$$

where

$$
C(f)=\frac{1}{(2 \pi f)^{2}+\tau^{-2}} \cdot \mathcal{A}^{-1}(f) \mathcal{B}\left(\mathcal{A}^{\dagger}(f)\right)^{-1}
$$

and the system matrix is

$$
-\mathcal{A}(f)=\mathcal{M}(2 \pi f)^{2}+j 2 \pi \mathcal{D} f+\mathcal{K} .
$$

The value of the integral (6) is determined by the singularities of the integrand in the complex plane of $f$, which in particular include zeros of the determinants $\operatorname{det} \mathcal{A}(f)$ and $\operatorname{det} \mathcal{A}(-f)$ as well as poles of $B(f)$ at $f= \pm(2 \pi \tau)^{-1}$. When the autocorrelation function of the system state vector in the frequency domain is analyzed, the contribution of the the poles of $B(f)$ can be filtered out by a high-pass filter. In time domain one can study the autostructure function $S\left(\left|t-t^{\prime}\right|\right)$ instead of $C\left(\left|t-t^{\prime}\right|\right)$, where the large contribution of the pole of $B(f)$ is cancelled out.

Naturally, among the remaining singularities, the one closest to the real $f$ axis undermines behavior of the autostructure function (6) at large $\delta t$. To identify this singularity, we recall that near a co-dimension 1 saddle node bifurcation one (and only one) of the eigenvalues of the power flow Jacobian $\mathcal{K}$ vanishes [20]. Close to the bifurcation, the inverse of the power flow Jacobian can be written as

$$
\mathcal{K}^{-1}=\frac{1}{\epsilon} b a^{T}+\tilde{\mathcal{K}}^{-1} \approx \frac{1}{\epsilon} b a^{T},
$$

where $\epsilon$ is the eigenvalue of $\mathcal{K}$ vanishing at the bifurcation, while $a$ and $b$ are the corresponding left and right eigenvectors.

Under these assumptions, the leading singularity of the integrand in (6) coincides with a zero of $\operatorname{det} \mathcal{A}(f)$ (or $\operatorname{det} \mathcal{A}(-f)$ depending on the sign of the difference $\left.t-t^{\prime}\right)$. Such singularity is a simple pole by assumption that the center manifold of the power system is one-dimensional. Constructing perturbation theory in powers of small $\epsilon$ and assuming the overdamped operating regime, one finds that the dominating mode determining behavior of the expectation value (6) at $\left|t-t^{\prime}\right| \rightarrow \infty$ is given by

$$
2 \pi f_{\text {corr }}=-\frac{j \epsilon}{a^{T} \mathcal{D} b}
$$

to the leading order in $\epsilon$. The $\mathcal{O}\left(\epsilon^{2}\right)$ contribution can be neglected as long as $\epsilon \ll\left(a^{T} \mathcal{D} b\right)^{2} /\left(a^{T} \mathcal{D}^{T} \tilde{\mathcal{K}}^{-1} \mathcal{D} b-a^{T} \mathcal{M} b\right)$. Note that the dependence of the leading mode on the matrix
$\mathcal{M}$ of inertia constants appears only in the second order in $\epsilon$ and is negligible in the vicinity of the bifurcation point, as $\epsilon \rightarrow 0$. The frequency of the leading mode is purely imaginary to the order $\mathcal{O}(\epsilon)$ unless $\epsilon>\left(a^{T} \mathcal{D} b\right)^{2} / 4 a^{T} \mathcal{M} b$, i.e., when the system is underdamped.

Estimating the integral (6) near the leading singularity $f=$ $f_{\text {corr }}$, one finally finds to the leading order in $\epsilon$

$$
\begin{gathered}
S(|\delta t|)=\frac{b\left(a^{T} B a\right) b^{T}|\delta t|}{\epsilon^{2}}+ \\
\frac{b\left(a^{T} B a\right)\left(a^{T} D b\right) b^{T}}{\epsilon^{3}}\left(e^{-\frac{\epsilon|\delta t|}{a^{T} D b}}-1\right) .
\end{gathered}
$$

Note that the matrix elements $a^{T} \mathcal{B} a$ and $a^{T} \mathcal{D} b$ are simple numerical factors, so that the dominating direction in the phase space of the system where the fluctuations of the system vector $x$ grow coincides with the direction of the right eigenvector $b$ corresponding to the lowest eigenvalue $\epsilon$ of the power flow Jacobian $\mathcal{K},[20],[21]$.

\section{DETECTING CRITICAL SLOWING-DOWN IN FREQUENCY AND TIME DOMAINS}

According to the expression (10), as $\epsilon \rightarrow 0$ and the operating regime of the grid approaches the bifurcation, amplitude of the fluctuations of the system state vector $x$ grows as $\epsilon^{-3}$. $^{2}$ Simultaneously, the characteristic correlation time of these fluctuations $\tau_{\text {corr }}=\frac{a^{T} D b}{\epsilon}$ grows as $\epsilon^{-1}$ at $\epsilon \rightarrow 0$. These two effects explain the phenomenon of critical slowing-down often observed during large-scale failures of power grids [11], [12], [13], [14]. The growth of both the amplitude of fluctuations and the correlation time imply that close to collapse dynamics of the system vector $x$ can be represented as a sequence of relatively long time intervals with weakly changing values of system variables $x$. However, these values significantly deviate from the equilibrium ones, $x_{0}$, determined by the stationarity condition $\dot{x}_{0}=0$. This in turn (at least partially) explains why it is technically hard to correctly and rapidly identify the state of the power system close to an unstable regime using the standard power flow estimated procedures [4], [5]: away from the threshold of instability stochastic fluctuations of the system state vector $x$ are suppressed, while close to this threshold they are strongly amplified. The same applies to numerical errors of power flow estimators in operating regimes near bifurcation and prevents effective convergence of numerical schemes. It is very tempting to use the very signatures of critical slowingdown as markers of approach to stability loss [11], [12], [13], [14]. In this Section we shall consider how the critical slowingdown affects behavior of the autocorrelation function in the frequency domain and then compare our conclusions to results of numerical simulations.

The complete dominance of a single mode close to collapse implies that in the frequency domain expression (7) reduces

\footnotetext{
${ }^{2}$ Note that the actual behavior of the amplitude of fluctuations as a function of $\epsilon$ depends on the load model, in particular, on the dependence of the expectation value of fluctuating loads on frequency $f\left(\sim f^{-2}\right.$ in our case).
} 
to

$$
C(f)=\frac{2 b\left(a^{T} \mathcal{B} a\right) b^{T}}{(2 \pi f)^{2}\left(\left(a^{T} \mathcal{D} b\right)^{2}(2 \pi f)^{2}+\epsilon^{2}\right)}+\mathcal{O}(\epsilon) .
$$

Thus, at very small angular frequencies $2 \pi f \ll \frac{\epsilon}{a^{T} \mathcal{D} b}$ one finds a quadratic behavior of the Fourier-transformed autocorrelation function (7) with $f^{-1}: S(f) \approx \frac{2 b\left(a^{T} \mathcal{B} a\right) b^{T}}{(2 \pi f \epsilon)^{2}}$, while in the intermediate range of frequencies this behavior is quartic instead: $S(f) \approx \frac{2 b\left(a^{T} \mathcal{B} a b^{T}\right.}{\left(a^{T} \mathcal{D} b\right)^{2}(2 \pi f)^{4}}$. These two types of behavior are rather universal and can be seen even if the dynamics of the power system does not yet exhibit critical slowing-down. Closer to the threshold of instability, as $\epsilon \rightarrow 0$, the quadratic behavior of (11) is realized in a smaller and smaller range of frequencies $\tau^{-1}<2 \pi f<\frac{\epsilon}{a^{T} \mathcal{D} b}$. This in turn leads to a stronger $1 / f^{4}$ amplification of the amplitude of fluctuations at small $f$ : the matching point $2 \pi f \sim \frac{\epsilon}{a^{T} \mathcal{D} b}$ between $f^{-4}$ and $f^{-2}$ regimes is reached at smaller and smaller $f$, while $\epsilon \rightarrow 0$. Corresponding amplification of the amplitude of fluctuations of $x$ particularly noticeable at small frequencies is a very good marker of approach to stability loss.

In order to check these predictions, we have performed numerical simulations of the stochastic behavior of IEEE 39 and IEEE 57 test power systems close to the threshold of instability in both frequency and time domains. Bifurcation points for both power systems discussed here were first localized using continuation power flow procedure [22], [23] implemented in PSAT Toolbox for Matlab [24] and then identified more precisely using MATPOWER library for Matlab [25].

For the case of the IEEE 39 test power system two chosen values of the continuation power flow parameter corresponded to the smallest eigenvalue of the power flow Jacobian $\epsilon \approx 0.57$ p.u. (operating regime relatively far from the threshold of instability) and $\epsilon \approx 0.08$ p.u. (pre-critical operating regime).The loads were allowed to fluctuate only on the buses 3,10 and 21 in order to check the difference in correlations of the state vector between the nodes with and without fluctuating power loads. On each of these nodes, a single realization of the active power load fluctuations was considered (although realizations of $\delta P_{i}$ of course differed between the nodes). Other parameters of the model were chosen similarly to [26], [13], which brings the system into an overdamped operating regime.

For the case of the IEEE 57 test power system the operational regimes chosen for simulations corresponded to $\epsilon \approx$ 0.01 p.u. (pre-critical regime) and $\epsilon \approx 0.17$ p.u. (normal stable operating regime). Parameters $\alpha_{i}$ were chosen as described in the Section 2. Inertia constants $H_{i}$ of generators were chosen according to the relation $H_{i} \approx 0.04 P_{i}$, which, as explained in [27], effectively holds for many test power system models. As usual, the bus 1 was the slack bus. The active power loads were allowed to fluctuate only on the nodes $12,28,45$ with characteristic amplitude of fluctuations $\sqrt{\mathcal{B}} /(2 \pi) \approx 0.1$ p.u.

The results of frequency domain simulations for the IEEE 39 model are presented on the Figs.2, 3. The $1 / f^{4}$ behavior along the relevant interval of frequencies is clearly seen for both cases of $\epsilon \approx 0.57$ p.u. and 0.08 p.u., as well as an amplification of the fluctuations at small frequencies by more than an order of magnitude in the pre-critical operating regime. The prediction of the theory for the value of the autocorrelation function at $f \approx 0.01 \mathrm{~Hz}$ is $\approx 3$ for the case $\epsilon \approx 0.08$ p.u. and $\approx 0.1$ for the case $\epsilon \approx 0.57$ p.u., in a good agreement with the results of simulation.

As expected, we have found that the low-frequency behavior of different inter-node/single-node structure functions of the system state vector $x$ is rather similar irrespective whether the power load is fluctuating or fixed on the given node. This implies that a single-node autocorrelation function of the system state vector, in particular, its behavior at small $f$, can be a good indicator of the approach to the loss of stability. The behavior at large frequencies differed noticeably, with internode correlations decaying more rapidly with $f$ for nodes with fixed power loads. The results of simulations of the IEEE 57 test power system are essentially similar.

The results of simulations of the autostructure function $S(|\delta t|)$ in time domain demonstate its amplification at $|\delta t| \sim$ $1-10 \mathrm{sec}$. The singular value decomposition of the system

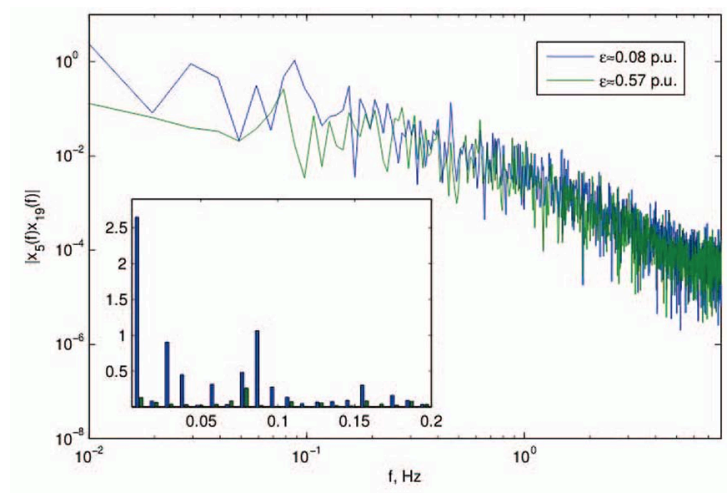

Fig. 2. Detecting critical slowing-down in frequency domain: simulations of IEEE 39. Inter-node correlation function $C\left(x_{5}(f) x_{19}(f)\right)$, the load on both nodes 5 and 19 . The regime $\epsilon \approx 0.08$ p.u. is close to the threshold of instability. while the regime with $\epsilon \approx 0.57$ p.u. is relatively far from the threshold. Amplification of the amplitude of fluctuations at small frequencies $f$ by more than an order of magnitude is clearly seen. The main plot uses the log-log scale. The inset represents behavior of the correlation function at small $f$ using the normal scale.

state vector $x(t)$ shows that there exists a dominating mode for the pre-critical regime $\epsilon \approx 0.01$ p.u., while this is not the case for the stable operating regime $\epsilon \approx 0.17$ p.u., where the contributions of many different modes to $x$ provide a contribution of the same order of magnitude into (6), see Fig. 4. Finally, we would like to emphasize that the contribution of different nodes into the right eigenvector $b$ of the leading mode relatively weakly depends on the operating regime. Thus, the dominant nodes of the grid contributing the most to this mode can be identified well before the threshold of instability is approached.

\section{CONCLUSION}

In the present contribution we have studied the phenomenon of critical slowing-down often observed in power grids close to 


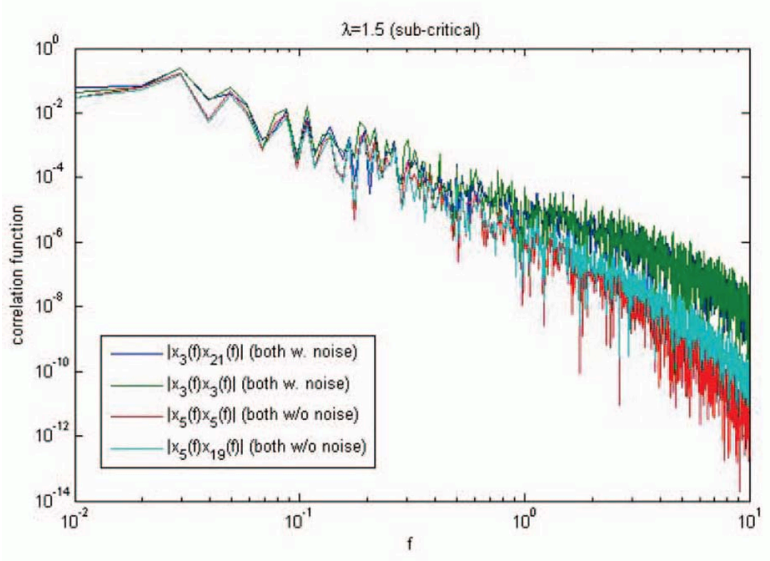

Fig. 3. Detecting critical slowing-down in the frequency domain; simulations of IEEE 39. Comparison of different inter-node and single-node structure functions in the operating regime close to the threshold of instability. As can be clearly seen, behavior of different inter-node structure functions is very similar at small frequencies. The log-log scale is used for the main plot. The inset represents behavior of the same correlation functions at small $f$ using the normal scale. The difference in amplitude of correlation functions at very small $f$ is fully explained by the difference in magnitudes of the corresponding components of the vector $b$.
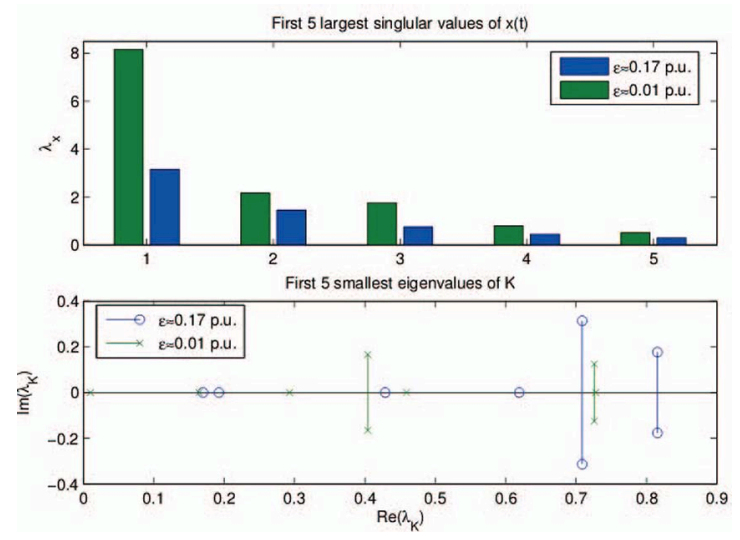

Fig. 4. 5 largest singular values of the system state vector $x$ of the IEEE 57 power system for pre-critical $(\epsilon \approx 0.01$ p.u.) and sub-critical $(\epsilon \approx 0.17$ p.u. $)$ operating regimes. 8 smallest eigenvalues of the power flow Jacobian for the same regimes.

the threshold of a large-scale failure such as voltage collapse or a loss of synchrony [11], [12], [13], [14]. As we have argued, this phenomenon is directly related to (and can be explained by) a strong amplification of fluctuations of the system state variables - voltage phases and magnitudes on individual nodes of the grid - for operating regimes close to collapse.

We have explained how the phenomenon of critical slowingdown can be used to effectively detect approach to the threshold of instability. A technically simple method of detection is based on analysis of single-node (or inter-node) autostructure functions of the system state vector in the frequency domain, where approach to the threshold of instability is characterized by a strong amplification of low frequency part of the correlation function. It is very important to note that the onset of critical slowing-down can be detected using limited measurements of state variables, such as in the situation of incomplete observability of the smart grid, when effective and precise measurement of inter-area modes is impossible.

\section{ACKNOWLEDGMENTS}

This work was partially supported by NSF award ECCS1128437 and MIT/SkTech seed funding grant.

\section{REFERENCES}

[1] G. Andersson, P. Donalek, R. Farmer, N. Hatziargyriou, I. Kamwa, P. Kundur, N. Martins, J. Paserba, P. Pourbeik, J. Sanchez-Gasca, R. Schulz, A. Stankovic, C. Taylor, and V. Vittal, "Causes of the 2003 Major Grid Blackouts in North America and Europe, and Recommended Means to Improve System Dynamic Performance," IEEE Transactions on Power Systems, vol. 20, no. 4, pp. 1922-1928, Nov. 2005.

[2] C. et al. Canizares, "Voltage Stability Assessment: Concepts, Practices and Tools," IEEE/FES Power System Stability Subcommittee, Technical Report, 2002.

[3] P. Pourbeik, P. Kundur, and C. Taylor, "The anatomy of a power grid blackout - Root causes and dynamics of recent major blackouts," IEEE Power and Energy Magazine, vol. 4, no. 5, pp. 22-29, Sep. 2006.

[4] F. Wu, "Theoretical study of the convergence of the fast decoupled load flow," IEEE Transactions on Power Apparatus and Systems, vol. 96, no. 1, pp. 268-275, Jan. 1977.

[5] R. Klump and T. Overbye, "Techniques for improving power flow convergence," in 2000 Power Engineering Society Summer Meeting (Cat. No.00CH37134), vol. 1. IEEE, 2000, pp. 598-603.

[6] K. Loparo and G. Blankenship, "A probabilistic mechanism for small disturbance instabilities in electric power systems," IEEE Transactions on Circuits and Systems, vol. 32, no. 2, pp. 177-184, Feb. 1985.

[7] C. Nwankpa, S. Shahidehpour, and Z. Schuss, "A stochastic approach to small disturbance stability analysis," IEEE Transactions on Power Systems, vol. 7, no. 4, pp. 1519-1528, 1992.

[8] M. Anghel, K. Werley, and A. Motter, "Stochastic Model for Power Grid Dynamics," in 2007 40th Annual Hawaii International Conference on System Sciences (HICSS'07). IEEE, 2007, pp. 113-113.

[9] Z. Y. Dong, J. H. Zhao, and D. J. Hill, "Numerical Simulation for Stochastic Transient Stability Assessment," IEEE Transactions on Power Systems, vol. 27, no. 4, pp. 1741-1749, Nov. 2012.

[10] T. Odun-Ayo and M. L. Crow, "Structure-Preserved Power System Transient Stability Using Stochastic Energy Functions," IEEE Transactions on Power Systems, vol. 27, no. 3, pp. 1450-1458, Aug. 2012.

[11] P. Hines, E. Cotilla-Sanchez, and S. Blumsack, "Topological Models and Critical Slowing down: Two Approaches to Power System Blackout Risk Analysis," in 2011 44th Hawaii International Conference on System Sciences. IEEE, Jan. 2011, pp. 1-10.

[12] E. Cotilla-Sanchez, P. Hines, and C. Danforth, "Predicting Critical Transitions from Time Series Synchrophasor Data," IEEE Transactions on Smart Grid, vol. 3, no. 4, pp. 1832-1840, 2012.

[13] D. Podolsky and K. Turitsyn, "Random load fluctuations and collapse probability of a power system operating near codimension 1 saddle-node bifurcation," in IEEE Power and Energy Society General Meeting (PES), 2013, pp. pp. 1-5.

[14] G. Ghanavati, P. D. H. Hines, T. Lakoba, and E. Cotilla-Sanchez, "Calculation of the autocorrelation function of the stochastic single machine infinite bus system," in 2013 North American Power Symposium (NAPS). IEEE, Sep. 2013, pp. 1-6.

[15] A. Bergen and D. Hill, "A Structure Preserving Model for Power System Stability Analysis," IEEE Transactions on Power Apparatus and Systems, vol. PAS-100, no. 1, pp. 25-35, Jan. 1981.

[16] P. Kundur, N. J. Balu, and M. G. Lauby, Power system stability and control. McGraw-Hill, 1994.

[17] D. Cox and H. Miller, The Theory of Stochastic Processes. Taylor \& Francis, 1977.

[18] J. F. Hauer, D. J. Trudnowski, and J. G. DeSteese, "A Perspective on WAMS Analysis Tools for Tracking of Oscillatory Dynamics," in 2007 IEEE Power Engineering Society General Meeting. IEEE, Jun. 2007. pp. $1-10$. 
[19] K. Wang and M. L. Crow, "The Fokker-Planck Equation for Power System Stability Probability Density Function Evolution," IEEE Transactions on Power Systems, vol. 28, no. 3, pp. 2994-3001, Aug. 2013.

[20] I. Dobson, "Observations on the geometry of saddle node bifurcation and voltage collapse in electrical power systems," IEEE Transactions on Circuits and Systems I: Fundamental Theory and Applications, vol. 39, no. 3, pp. 240-243, Mar. 1992.

[21] C. Canizares, "On bifurcations, voltage collapse and load modeling," IEEE Transactions on Power Systems, vol. 10, no. 1, pp. 512-522, 1995

[22] V. Ajjarapu and C. Christy, "The continuation power flow: a tool for steady state voltage stability analysis," IEEE Transactions on Power Systems, vol. 7, no. 1, pp. 416-423, 1992.

[23] R. Avalos, C. Canizares, F. Milano, and A. Conejo, "Equivalency of Continuation and Optimization Methods to Determine Saddle-Node and Limit-Induced Bifurcations in Power Systems," IEEE Transactions on Circuits and Systems I: Regular Papers, vol. 56, no. 1, pp. 210-223, Jan. 2009.

[24] F. Milano, "An Open Source Power System Analysis Toolbox," IEEE Transactions on Power Systems, vol. 20, no. 3, pp. 1199-1206, Aug. 2005.

[25] R. D. Zimmerman, C. E. Murillo-Sanchez, and R. J. Thomas, "MATPOWER: Steady-State Operations, Planning, and Analysis Tools for Power Systems Research and Education," IEEE Transactions on Power Systems, vol. 26, no. 1, pp. 12-19, Feb. 2011.

[26] M. Pai, Energy Function Analysis for Power System Stability. Springer, 1989.

[27] A. E. Motter, S. A. Myers, M. Anghel, and T. Nishikawa, "Spontaneous synchrony in power-grid networks," Nature Physics, vol. 9, no. 3, pp. 191-197, Feb. 2013. 